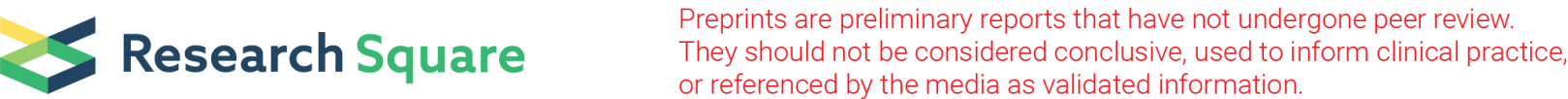

\section{Quantitative CT analysis to predict esophageal fistula in patients with advanced esophageal cancer treated by chemotherapy or chemoradiotherapy}

Ying-Shi Sun ( $\sim$ sys27@163.com )

Peking University Cancer Hospital

Yan-Jie Shi

Peking University Cancer Hospital

Chang Liu

Peking University Cancer Hospital

Yi-Yuan Wei

Peking University Cancer Hospital

Xiao-Ting Li

Peking University Cancer Hospital

Lei Tang

Peking University Cancer Hospital

Lin Shen

Peking University Cancer Hospital

Zhi-Hao Lu

Peking University Cancer Hospital

\section{Research Article}

Keywords: esophageal squamous cell carcinoma, X-ray computed Tomography, esophageal fistula, risk factor, chemoradiotherapy

Posted Date: February 21st, 2022

DOI: https://doi.org/10.21203/rs.3.rs-742527/v2

License: (c) (i) This work is licensed under a Creative Commons Attribution 4.0 International License.

Read Full License 


\section{Abstract}

Background: Esophageal fistula is one of the most serious complications of chemotherapy or chemoradiotherapy (CRT) for advanced esophageal cancer. This study aimed to evaluate the performance of quantitative computed tomography (CT) analysis and to establish a practical imaging model for predicting esophageal fistula in esophageal cancer patients administered chemotherapy or chemoradiotherapy.

Methods: This study retrospectively enrolled 204 esophageal cancer patients ( 54 patients with fistula, 150 patients without fistula) and all patients were allocated to the test and validation cohorts according to the time of inclusion in a 1:1 ratio. Ulcer depth, tumor thickness and length, and minimum and maximum enhanced values for esophageal cancer were measured in pretreatment CT imaging. Logistic regression analysis was used to evaluate the associations of CT quantitative measurements with esophageal fistula. Receiver operating characteristic curve (ROC) analysis was also used.

Results: Logistic regression analysis showed that independent predictors of esophageal fistula included tumor thickness [odds ratio $(\mathrm{OR})=1.167 ; p=0.037$ ], the ratio of ulcer depth to adjacent tumor thickness $(\mathrm{OR}=164.947 ; p<0.001)$, and the ratio of minimum to maximum enhanced CT value $(\mathrm{OR}=0.006 ; p=$ 0.039) in the test cohort at baseline CT imaging. These predictors were used to establish a predictive model for predicting esophageal fistula, with areas under the receiver operating characteristic curves (AUCs) of 0.946 and 0.841 in the test and validation groups, respectively.

Conclusions: Quantitative pretreatment CT analysis has excellent performance for predicting fistula formation in esophageal cancer patients who treated by chemotherapy or chemoradiotherapy.

\section{Background}

Latest cancer statistics revealed that esophageal cancer is an extremely devastating disease among the 10 most common malignancies causing death [1]. According to the Comprehensive Registry of Esophageal Cancer in Japan, the incidence of T4b disease represents approximately $9 \%$ of all thoracic esophageal cancer [2]. The incidence of distant metastases is approximately $40 \%$ among all esophageal cancer patients [1]. Curative resection is not feasible in esophageal cancer patients with T4b or distant metastasis, who have unfavorable prognosis [2]. Chemotherapy or chemoradiotherapy (CRT) without planned esophagectomy is one of the most attractive treatment options available for unresectable esophageal cancer [3]. Previous reports indicated that chemotherapy or CRT could allow $32-60 \%$ of patients to undergo curative resection for $\mathrm{cT} 4 \mathrm{~b}$ esophageal cancer, suggesting that curative resection has a good prognosis [4-6].

However, esophageal fistula represents one of the known serious complications of esophageal cancer, especially in cases treated by chemotherapy or CRT. Chemotherapy or CRT could induce esophageal fistula by damaging the walls of the esophagus and adjacent organs. The imbalance between the treatment response of the tumor and normal tissue repair may lead to esophageal fistula [7-9]. Although 
the incidence of esophageal fistula is low with a range of $10.4 \%-13.9 \%$, it is potentially life-threatening with high mortality rates of $10-25 \%[10,11]$. It is generally admitted that mortality could at least double when the diagnostic and therapeutic delay exceeds 24 hours [12]. Therefore, esophageal fistula could not only result in horrible life quality, but also change the therapeutic effect and clinical management.

Thus, predicting esophageal fistula occurrence and identifying the associated high-risk factors is a major clinical problem before chemotherapy or CRT for esophageal carcinoma. Various risk factors have been considered key factors associated with esophageal fistula formation in patients with esophageal cancer after treatment, e.g., esophageal stenosis, nutritional status, body mass index (BMI), tumor length, presence of ulcer in the tumor and T stage [13-15] .

Currently, chest computed tomography (CT), endoscopic ultrasound (EUS) and esophagography are the main imaging tools for evaluating esophageal cancer. Esophagography and EUS are limited in evaluating advanced esophageal tumors whose outer borders might be outside the field of view, especially stenotic tumors [16]. Endoscopy and EUS are direct and effective methods, but both highly operator-dependent, could damage the esophagus, which may lead to esophageal fistula in high risk patients with suspicious esophageal fistula. Meanwhile, chest CT is inexpensive, easy to perform and reproducible, and could provide morphological and quantitative information for the lesions and surrounding conditions [17]. Therefore, CT may provide useful information for predicting esophageal fistula before treatment.

To our knowledge, the role of CT features in predicting esophageal fistula had been rarely reported. Therefore, this retrospective study aimed to identify risk factors for esophageal fistula, to investigate the performance of quantitative CT analysis and to develop a practical imaging model in predicting the risk of esophageal fistula before treatment in patients with esophageal cancer.

\section{Methods}

\section{Patients}

Inclusion criteria were: a) fibergastroscopic biopsy-proven esophageal squamous cell carcinoma (SCC) before treatment; $b$ ) chest enhanced CT examination at baseline; $c$ ) chemotherapy or CRT; d) diagnosis with esophageal fistula by CT, endoscopy, barium esophagography or operation at follow-up; e) availability of quality diagnostic images for measuring lesions. Patients who were not in accord with inclusion criteria were excluded. Patients with esophageal cancer accompanied by esophageal fistula after chemotherapy or CRT were identified from January 2011 to December 2019. In addition, esophageal SCC patients with no esophageal fistula after chemotherapy or CRT were included in this study from January 2016 to December 2017 in a 1:3 ratio. The patients were allocated to the test cohort and validation cohorts in a 1:1 ratio according to the time of inclusion. The complete patient enrollment process is shown in Figure 1.

\section{Chemotherapy or CRT}


Patients treated with chemotherapy received a platinum-based regimen, mainly including paclitaxel (175 $\mathrm{mg} / \mathrm{m} 2$ i.v. on day 1 of every 3-week cycle) and cisplatin ( $75 \mathrm{mg} / \mathrm{m} 2$ i.v. on day 1 of every 3-week cycle). Other patients were treated with concurrent chemoradiotherapy, with a radiotherapy dose of 50-60Gy and platinum-based chemotherapy.

\section{Computed tomography}

All patients received enhanced multi-slice CT (MDCT) scanning of the chest before and during chemotherapy or CRT. Scans were performed using a 64-row helical CT scanner (Lightspeed VCT; General Electrical Medical Systems, Milwaukee, WI, USA). All patients were in the supine position. Generally, the scan began at $2.0 \mathrm{~cm}$ above the lung apices and extended through the adrenal glands. The following imaging parameters were used: $120-140 \mathrm{kVp}$ tube voltage; $300 \mathrm{~mA}$ tube current; $64 \times 0.625 \mathrm{~mm}$ detector collimation; $0.6 \mathrm{~s} /$ rotation gantry rotation speed, and 1.5 helical pitch. Axial, coronal and sagittal images were reconstructed using a section width of $5.0 \mathrm{mmOne}$ hundred milliliters of the non-ionic contrast medium iohexol (Omnipaque 300; GE Healthcare) was injected at a rate of $3.0 \mathrm{~mL} / \mathrm{s}$ through the median cubital vein.

\section{Image Interpretation}

MDCT imaging data were transferred to a picture archiving and communication system (PACS). Two radiologists with 8 years (Dr. Wei) and 12 years (Dr. Shi) of experience in thoracic CT independently reviewed the axial and reconstructed CT images obtained at baseline. Both reviewers were blinded to final results about esophageal fistula. All qualitative and quantitative parameters were assessed on enhanced images before treatment. Final quantitative measurements were determined by averaging the values obtained by the two radiologists. For qualitative analysis, the diagnosis was confirmed by a third experienced radiologist in case of disagreement.

\section{Qualitative analysis}

Tumor locations were classified as cervical, upper thoracic, middle thoracic, or lower thoracic esophagus. An important factor in assessing cancer location was to determine the center of the tumor in the esophagus. The tumor was staged by MDCT before therapy according to AJCC/TNM classification, 8th edition. The MDCT status was defined as follows [10] : CT T0, wall thickness $<5.0 \mathrm{~mm}$ and no signs of adventitial penetration; CT T1-2, a wall thickness of at least $5-10 \mathrm{~mm}$ without evidence of adventitial penetration; CT T3, tumor exhibiting a wall thickness of $>10 \mathrm{~mm}$, possibly appearing as ill-defined, abnormal soft tissue around the tumor but no invasion of adjacent structures; CT T4a, invasion of the pleura, pericardium and diaphragm; $\mathrm{T} 4 \mathrm{~b}$, invasion of the aorta, vertebral body and trachea. In the last two stages, the tumor had a wall thickness of $>10 \mathrm{~mm}$ and invaded adjacent structures. Intrathoracic and abdominal lymph nodes $>10 \mathrm{~mm}$ and supraclavicular lymph nodes $>5.0 \mathrm{~mm}$ in short-axis diameter were 
considered metastatic lymph nodes [18] . N staging was classified as negative (N-) or positive $(\mathrm{N}+)$ metastatic lymph nodes. CT imaging findings related to tracheal or bronchial invasion of the tumor were analyzed. The tumor range was classified as four types, including $0-1 / 4,1 / 4-1 / 2,1 / 2-3 / 4$ and 3/4-1 (Figure 2). Esophageal stenosis and deep ulcer were also evaluated. The morphological patterns of the tumor were graded as focal or diffuse.

\section{Quantitative analysis}

Tumor wall thickness (THK-tumor) and thinness (THN-tumor) of esophageal SCC were measured perpendicularly to the lumen on axial images using the workstation's electronic caliper. In case of invisible lumen, the maximal tumor diameter was obtained and multiplied by 0.5 . Tumor length (the tumor's longest diameter, L-tumor) was measured on sagittal CT images. The region of interest (ROI) of the tumor's maximum CT value (HU-max) was placed on the highly enhanced area and that of the tumor's minimum CT value (HU-min) on the lowly enhanced area (Figure 3). The area of each ROI was 3-5 mm², averaging three measurements. The depths of low and high intensity enhancement areas were also measured (Figure 3). Tumor ulceration was quantitatively assessed by measuring ulcer depth (DEP-ulcer), the thickness of the residual wall in the ulcer layer (THK-residue), and the thickness of the lesion adjacent to the ulcer (THK-adjacency) on cross-sectional CT images (Figure 2, 3). In case of no ulcer in the tumor, DEP-ulcer was recorded as 0 , and THK-residue and THK-adjacency were the same as the wall thickness of the tumor. The ulcer-to-tumor ratio (R-ulcer) was calculated by the following equation: DEP-ulcer/THKadjacency. The THK-residue-to-tumor ratio (R-residue) was derived as THK-residue/THK-adjacency. The HU-min to HU-max (R-HU) ratio was obtained as HU-min/HU-max. The joint predictive efficiency of continuous variables was defined as $\mathrm{Y} 1$. Y1 combining qualitative signs was defined as $\mathrm{Y} 2$.

\section{Definition of esophageal fistula}

Esophageal fistula was defined as a connection between the esophagus and adjacent organs or tissues [19] detected by CT, endoscopy, barium esophagography or operation. On CT images, esophageal fistula was diagnosed by discontinuous or defective esophageal wall, gas and fluid accumulated around the esophagus, or pneumonia associated with esophageal fistula. By esophagography, esophageal fistula was identified as contrast medium leakage into the mediastinum or bronchus.

\section{Statistical Analysis}

Differences in qualitative parameters in patients with esophageal SCC between the test and validation cohorts were assessed by the Mann-Whitney test or the Chi-square test/Fisher's exact test. Differences in quantitative factors were examined by independent-samples $t$ test or the Mann-Whitney test. The associations of quantitative measurements were evaluated by Pearson correlation coefficient; a coefficient $>0.6$ suggested a moderate or strong correlation. Only parameters with weak correlation were 
substituted into the multivariate equation. Receiver operating characteristic (ROC) curve analysis was applied to evaluate the predictive capability of the quantitative analysis for predicting esophageal fistula, with the area under the ROC curve (AUC). Intraclass correlation coefficients (ICCs) were determined to evaluate inter-observer agreement in terms of parameter extraction. Data analysis was conducted with SPSS 22.0 (IBM Corporation, Armonk, NY, USA) and STATA 12.0 (Stata Corporation, College Station, TX, USA).

\section{Results}

\section{Clinical characteristics of the patients}

A total of 204 patients were enrolled in this study. Totally 54 patients with esophageal fistula were identified. In parallel, 150 esophageal SCC patients with non-esophageal fistula after treatment were included in this study. The clinical characteristics of the patients are summarized in Table 1. There were no significant differences in clinical characteristics between the test and validation cohorts (See Table 2). Two radiologists independently assessed esophageal SCC, achieving satisfactory agreement. Qualitative and quantitative analyses between the two radiologists showed perfect or substantial agreement with ICCs of $0.788-0.896$.

\section{Univariable comparisons of CT's quantitative parameters}

Table 1 shows the univariable comparisons of CT's qualitative and quantitative parameters for predicting fistula in the test and validation cohorts. The qualitative analysis showed that middle thoracic location, T4b staging, trachea invasion, tumor range of 3/4-1, tumor location in the middle thoracic esophagus and ulcer presence were risk factors for esophageal fistula before treatment $(P<0.05)$. There were significant differences in tumor location, range, and presence of deep ulcers between the fistula and non-fistula groups in qualitative analysis $(P<0.001)$. In CT's quantitative analysis, tumor thickness, tumor length, depth of the ulcer and ulcer ratio in the fistula group were larger than those of the non-fistula group $(P<0.001)$. HU-min, ratio of HU-min to HU-max, and tumor thinness were smaller in the fistula group compared with the non-fistula group in the test and validation cohorts $(P<0.05)$.

\section{Predictive performance of CT's quantitative analysis}

Correlation analyses of parameters obtained in quantitative CT analysis revealed that the correlation coefficients for HU-min and HU-min to HU-max ratio, THK-tumor and THK-adjacency, and DEP-ulcer and Rulcer were $>0.6$. HU-min to HU-max ratio, THK-tumor and R-ulcer were used in logistic regression models for predicting esophageal fistula due to high AUCs.

This logistic regression analysis revealed that esophageal cancer with high R-ulcer had elevated predicted rate of esophageal fistula [odds ratio $(\mathrm{OR})=164.947$; $95 \%$ confidence interval $(\mathrm{Cl})$ : 20.464- 
1329.511]. Esophageal cancer with lower R-HU value was associated with higher rate of esophageal fistula after treatment ( $\mathrm{OR}=0.006 ; 95 \% \mathrm{Cl}$ : 0.001-0.782). Esophageal cancer with higher THK-tumor was also correlated with higher rate of esophageal fistula (OR=1.167; 95\% Cl: 1.009-1.351) (Table 3). R-ulcer had the highest performance with an AUC of $0.887(95 \% \mathrm{Cl} 0.800-0.975)$ for predicting esophageal fistula, followed by R-HU with an AUC of $0.774(95 \% \mathrm{Cl} 0.677-0.872)$ and THK-tumor with an AUC of $0.696(95 \% \mathrm{Cl}$ 0.582-0.809) (Table 3).

So, R-ulcer, R-HU and THK-tumor were used for establishing logistic regression models due to high performance in predicting esophageal fistula at baseline CT. This quantitative CT model (using the formula $\mathrm{Y} 1=0.155^{*} \mathrm{THK}$-tumor+5.106*R-ulcer-5.105*R-HU) for predicting esophageal fistula had great performance with an AUC of $0.946(95 \% \mathrm{Cl} 0.902-0.990)$ and an accuracy of $92.2 \%$ in the test cohort. The same high performance was found in the validation cohort with an AUC of $0.841(95 \% \mathrm{Cl} 0.758-0.924)$ and an accuracy of $78.4 \%$ in predicting esophageal fistula. Detailed information of the efficiency in predicting esophageal fistula in the test and validation cohorts was shown in Table 4. The predictive capabilities of quantitative CT model for esophageal fistula in the test and validation cohorts were determined by ROC curve analysis (Figure 4).

\section{Performance of the combination of CT's quantitative and qualitative analyses}

Ulcer presence in tumors had the highest performance in predicting esophageal fistula with an AUC of $0.874(95 \% \mathrm{Cl} 0.782-0.996)$, followed by tumor range with an AUC of $0.756(95 \% \mathrm{Cl}: 0.660-0.853)$ and T stage with an AUC of 0.726 (95\% Cl: 0.600-0.852) (See Table 5). However, only T stage was an independent factor $(p=0.024)$ in multivariate analysis. Therefore, $T$ stage and quantitative analysis were combined to establish a model for predicting esophageal fistula in patients with esophageal cancer. The combination model $\left(Y 2=0.935^{*} Y 1+2.033^{*} \mathrm{~T}\right.$-stage) for predicting esophageal fistula had an AUC of 0.953 $(95 \% \mathrm{Cl} 0.909-0.997)$ and an accuracy of $94.1 \%$ (Figure 3$)$. However, there was no statistical significance between quantitative CT and T stage combination and quantitative CT analysis ( $p>0.05)$.

\section{Clinical usefulness}

To facilitate clinical use, a nomogram based on quantitative CT parameters, including THK-tumor, R-ulcer and R-HU, was developed (Figure 5). The probability of fistula formation after treatment ranged from 0 to 1. A probability nearing 1 indicated high odds of esophageal fistula. Patients with esophageal cancer could benefit from this prediction model.

\section{Discussion}

This study showed that wall thickness in esophageal SCC was a significant risk factor for esophageal fistula formation. Wall thickness in esophageal SCC was associated with T stage. The current study also 
revealed that esophageal fistula was associated with deep ulcer presence and ulcer-to-tumor ratio.

The diagnosis of esophageal fistula after treatment is challenging. Direct imaging methods, including esophagography, CT and esophagoscopy, could provide important clues for the diagnosis of esophageal fistula. Esophagography performed with water soluble agents could detect $75 \%$ of thoracic fistulas [20]. However, it may produce false-negative results in $10-38 \%$ of patients because of aspired hypertonic oral contrast solution, which may promote pulmonary edema [21]. Most surgeons are concerned about barium extravasation into the thorax [21, 22]. A recent study showed that oral meglumine diatrizoate esophagography using CT has a high sensitivity of $100 \%$ and a specificity of $98.9 \%$ in esophageal fistula screening [23] .However, in $20 \%$ of cases, the fistula could not be identified by preoperative CT. Indeed, water-soluble contrast agents are hyperosmolar and could draw fluid into the lungs, causing pulmonary edema in case of aspiration into the tracheobronchial tree [21]. Esophagoscopy both detects the fistula and helps determine the method of treatment [22]. On the other hand, small fistulas may even escape the sight of experienced endoscopists. In addition, endoscopy may make the fistula bigger and create more contaminations [20].

Therefore, predicting esophageal fistula occurrence and selecting high risk patients for fistula in esophageal SCC before treatment may change the treatment strategy and help prevent such a complication after treatment. Previous studies investigated clinical risk factors associated with esophageal fistula formation in esophageal SCC [19]. However, clinical risk factors predicting esophageal fistula show moderate performance. In addition, no definitive factors in quantitative CT analysis have been identified in patients undergoing chemotherapy or CRT.

It was proved that T stage was a significant risk factor for fistula in accordance with previous results [24], which had a certain relationship with wall thickness in esophageal SCC. Another mentioned risk factors were deep ulcer presence and ulcer-to-tumor ratio in our study. Ulcerative lesions in esophageal carcinoma often destroy or penetrate the muscular layer, which may decrease the function of esophageal wall and increase the incidence rate of esophageal fistula in patients with increased pressure in the lumen because of swallowing or cough. Infection of ulcerative lesions also increases the risk of esophageal fistula. Sun et al.[25] found 11 patients (65\%) with deep ulcerative esophageal carcinoma among 17 patients who developed esophageal fistula. Another study by Tsushima et al.[14] demonstrated that $89 \%$ of patients with esophageal fistula presented ulcerative esophageal carcinoma. Hu et al.[24] indicated that ulcerative esophageal cancer was associated with esophageal fistula. The advantage of this study was that quantitative CT analysis assessing deep ulcers was more accurate in predicting esophageal fistula.

In the present study, there was a negative correlation between esophageal fistula and the ratio of minimum to maximum enhanced CT value. Tumor enhancement was positively correlated with density and micro-vessel structure in the tumor, which is the pathological basis of contrast-enhanced CT scanning [26]. Lower enhancement might reflect decreased intratumoral micro-vessel density, indicating less chance to absorb oxygen and nutrition as well as high odds of edema and necrosis. In Torres et al.' 
study, there was a positive correlation between intratumoral micro-vessel count and desmoplasia [27]. Most esophageal carcinomas showed moderate enhancement, which was lower than that of the inflammatory or fibrotic component. Higher enhancement might reflect inflammatory or fibrotic changes or heterogeneity within the tumor. It was speculated that the tumor area with elevated enhancement might be the active area of fibrous tissue proliferation. We supposed that necrosis in the tumor shows lower enhancement with reduced CT value which may develop fistula easily, while elevated CT value with active desmoplasia may prevent fistula formation in the esophagus. Consequently, we developed and validated a practical imaging scoring model for predicting high risk of esophageal fistula before treatment. This imaging scoring model could provide an effective and handy tool for clinical strategymaking.

There were several limitations in this study. First, the sample size was small due to the low incidence of esophageal fistula. A much larger database from large multicenter trials might address this shortcoming and validate the reproducibility and generalization of this model. Secondly, this was a retrospective study, with the inherent selection bias. A well-designed prospective trial comparing esophageal fistula incidence between patients with esophageal SCC with or without risk factors before treatment is warranted. Thirdly, this prediction model did not completely mitigate the subjective evaluation of radiologists, and might be also affected by radiologist experience.

\section{Conclusions}

We provide a handy and effective imaging model based on quantitative pretreatment CT parameters with excellent performance in predicting the risk of fistula formation in esophageal cancer patients administered chemotherapy or CRT. This model offers an individualized assessment approach for esophageal SCC patients to guide clinical treatment for esophageal cancer with high risk of esophageal fistula.

\section{Abbreviations}

DEP-ulcer: depth of deep ulcer

HU-max: tumor's maximum CT value

HU-min: tumor's minimum CT value

L-tumor: tumor length

R-HU: HU-min-to-HU-max ratio

R-min: THK-min-to-THK-tumor ratio

R-residue: THK-residue-to-tumor ratio 
R-ulcer: ulcer-to-tumor ratio

THK-adjacency: thickness of the lesion adjacent to the ulcer

THK-max: thickness of the tumor on the maximum CT value layer

THK-min: thickness of the tumor on the minimum CT value layer

THK-residue: thickness of residual esophageal wall in the ulcer layer

THK-tumor: tumor thickness

THN-tumor: tumor thinness

Y1: joint predictive efficiency of quantitative CT analysis

Y2: joint predictive efficiency of quantitative $\mathrm{CT}$ analysis combined with $\mathrm{T}$ stage

\section{Declarations}

\section{Ethical approval and consent to participate}

This study was approved by the Ethics Committee of Key Laboratory of Carcinogenesis and Translational Research (Ministry of Education), Peking University Cancer Hospital \& Institute (No: 2017KT11) and individual consent for this retrospective analysis was waived. The authors are accountable for all aspects of the work in ensuring that questions related to the accuracy or integrity of any part of the work are appropriately investigated and resolved. The study was conducted in accordance with the Declaration of Helsinki (was revised in 2013).

\section{Consent for publication}

Not applicable.

\section{Availability of data and materials}

The datasets used and/or analysed during the current study are available from the corresponding author on reasonable request.

\section{Competing of interest}

The authors declare that they have no competing interests. 


\section{Funding}

This work was supported by Beijing Natural Science Foundation [Z200015]; Beijing Municipal Administration of Hospitals Incubating Program [Code.PX2020046]; The third round of public welfare development and reform pilot projects of Beijing Municipal Medical Research Institutes [Beijing Medical Research Institute, 2019-1]; 2019 SKY Imaging Research Fund of the Chinese International Medical Foundation [Project No.Z-2014-07-1912]; National Natural Science Foundation of China [81971584】 91959116]; Beijing Municipal Administration of Hospitals Clinical Medicine Development of Special Funding Support [No.ZYLX201803]; 'Beijing Hospitals Authority'Ascent Plan [Code.20191103]; Peking University-Baidu Fund [Grant No. 2020BD027].

\section{Authors' contributions}

YJS, CL and YYW contributed to the conception of the study and interpretation of data and drafted the manuscript. CL, ZHL and LS performed treatment. XTL and LT participated in the statistical analysis. YSS, LS and ZHL provided the administrative support. All authors approved the submitted manuscript and agreed both to be personally accountable for the author's own contributions and to ensure that questions related to the accuracy or integrity of any part of the work.

\section{Acknowledgements}

Not applicable.

\section{Author details}

${ }^{a}$ Key Laboratory of Carcinogenesis and Translational Research (Ministry of Education), Department of Radiology, Peking University Cancer Hospital \& Institute, No.52 Fu Cheng Road, Hai Dian District, Beijing, 100142, China

${ }^{b}$ Key Laboratory of Carcinogenesis and Translational Research (Ministry of Education), Department of Gastrointestinal Oncology, Peking University Cancer Hospital \& Institute, No.52 Fu Cheng Road, Hai Dian District, Beijing, 100142, China

\section{References}

1. Siegel RL, Miller KD, Jemal A. Cancer statistics, 2020. CA Cancer J Clin. 2020; 70(1):7-30.

2. Tachimori Y, Ozawa S, Numasaki $\mathrm{H}$, et al. Comprehensive registry of esophageal cancer in Japan, 2012. Esophagus 2019; 16(3):221-245. 
3. Shinoda M, Ando N, Kato K, et al. Japan Clinical Oncology Group. Randomized study of low-dose versus standard-dose chemoradiotherapy for unresectable esophageal squamous cell carcinoma (JCOG0303). Cancer Sci. 2015; 106(4):407-12.

4. de Manzoni G, Pedrazzani C, Pasini F, et al. Chemoradiotherapy followed by surgery for squamous cell carcinoma of the thoracic esophagus with clinical evidence of adjacent organ invasion. J Surg Oncol 2007; 95(3):261-6.

5. Pimiento JM, Weber J, Hoffe SE, et al. Outcomes associated with surgery for T4 esophageal cancer. Ann Surg Oncol 2013; 20(8):2706-12.

6. Sjoquist KM, Burmeister BH, Smithers BM, et al. Australasian Gastro-Intestinal Trials Group. Survival after neoadjuvant chemotherapy or chemoradiotherapy for resectable oesophageal carcinoma: an updated meta analysis. Lancet Oncol 2011; 12:681-692.

7. Gabrail NY, Harrison BR, Sunwoo YC. Chemo-irradiation induced aortoesophageal fistula. J Surg Oncol 2010; 48: 213-5.

8. Sivaraman SK, Drummond R. Radiation-induced aortoesophageal fistula: An unusual case of massive upper gastrointestinal bleeding. J Emerg Med 2002; 23: 175-8.

9. Han X, Zhao YS, Fang Y, et al. Placement of transnasal drainage catheter and covered esophageal stent for the treatment of perforated esophageal carcinoma with mediastinal abscess. J Surg Oncol 2016; 114: 725-30.

10. Biancari F, D'Andrea V, Paone R, et al.Current Treatment and Outcome of Esophageal Perforations in Adults: Systematic Review and Meta-Analysis of 75 Studies. World J Surg 2013; 37: 799.

11. Zhang Y, Li Z, Zhang W, et al. Risk factors for esophageal fistula in patients with locally advanced esophageal carcinoma receiving chemoradiotherapy. Onco Targets Ther 2018; 11: 2311-7.

12. Persson S, Rouvelas I, Irino T, et al. Outcomes following the main treatment options in patients with a leaking esophagus: a systematic literature review. Dis Esophagus 2017; 30(12):1-10.

13. Watanabe S, Ogino I, Kunisaki C, et al. Relationship between nutritional status and esophageal fistula formation after radiotherapy for esophageal cancer. Cancer Radiother 2019; 23(3):222227. DOI: 10.1016/j.canrad.2018.10.005

14. Tsushima T, Mizusawa J, Sudo K, Honma Y, et al. Japan Esophageal Oncology Group of Japan Clinical Oncology Group (JCOG). Risk Factors for Esophageal Fistula Associated With Chemoradiotherapy for Locally Advanced Unresectable Esophageal Cancer A Supplementary Analysis of JCOG0303.Medicine (Baltimore) 2016; 95(20):e3699.

15. Xu Y, Wang L, He B, et al. Development and validation of a risk prediction model for radiotherapyrelated esophageal fistula in esophageal cancer. Radiat Oncol 2019; 14(1):181.

16. Hong SJ, Kim TJ, Nam KB, et al. New TNM staging system for esophageal cancer: what chest radiologists need to know. Radiographics 2014; 34:1722-1740.

17. Garge S, Rao KL, Bawa M. The role of preoperative CT scan in patients with tracheoesophageal fistula: a review. J Pediatr Surg 2013; 48(9):1966-71. 
18. Konieczny A, Meyer P, Schnider A, et al. Accuracy of multidetector-row CT for restaging after neoadjuvant treatment in patients with oesophageal cancer. Eur Radiol. 2013; 23(9):2492-502.

19. Kawakami T, Tsushima T, Omae K, et al. Risk factors for esophageal fistula in thoracic esophageal squamous cell carcinoma invading adjacent organs treated with definitive chemoradiotherapy: a monocentric case-control study. BMC Cancer 2018; 18: 573.

20. Eroglu A, Turkyilmaz A, Aydin Y, et al. Current management of esophageal perforation: 20 years experience. Dis Esophagus 2009; 22:374-80.

21. Swanson JO, Levine MS, Redfern RO, et al. Usefulness of high-density barium for detection of leaks after esophagogastrectomy, total gastrectomy, and total laryngectomy. AJR 2003; 181:415-420.

22. Eroglu A, Aydin Y, Yilmaz O. Thoracic perforations-surgical techniques. Ann Transl Med 2018; 6(3):40.

23. Wu R, Geng L, Zhao Z, et al. Clinical Application of Oral Meglumine Diatrizoate Esophagogram in Screening for Esophageal Fistula During Radiotherapy or Chemoradiotherapy for Esophageal Cancer. Front Oncol 2020; 10: 562147.

24. Hu B, Jia F, Zhou H, et al. Risk Factors Associated with Esophageal Fistula after Radiotherapy for Esophageal Squamous Cell Carcinoma. J Cancer 2020;11(12):3693-3700.

25. Sun X, Han S, Gu F, et al. A retrospective comparison of taxane and fluorouracil-based chemoradiotherapy in patients with inoperable esophageal squamous cell carcinoma. J Cancer 2016; 7: 1066-73.

26. Miles KA. Tumor angiogenesis and its relation to contrast enhancement on computed tomorgraphy: a review. Eur J Radiol 1999; 30(3):198-205.

27. Torres $\mathrm{C}$, Wang $\mathrm{H}$, Turner $\mathrm{J}$, et al. Prognostic significance and effect of chemoradiotherapy on microvessl density(angiogenesis) in esophageal Barrett's esophagus-associated adenocarcinoma and squamous cell carcinoma. Hum Pathol 1999; 30(7):753-8.

\section{Tables}

\section{Table 1}

\section{Characteristics of patients in the test and validation cohorts}




\begin{tabular}{|c|c|c|c|c|c|c|}
\hline & Test cohort & & & Validation cc & & \\
\hline Characteristic & non-fistula & fistula & $P$ & non-fistula & fistula & $P$ \\
\hline Gender (\%) & & & 0.185 & & & 0.307 \\
\hline Male & 68 (90.67\%) & 27 (100\%) & & $69(92 \%)$ & 23 (85.19\%) & \\
\hline Female & $7(9.33 \%)$ & $0(0 \%)$ & & $6(8.0 \%)$ & $4(14.81 \%)$ & \\
\hline $\begin{array}{l}\text { Age, mean } \pm \\
\text { SD, years }\end{array}$ & $60.62 \pm 8.05$ & $59.07 \pm 7.26$ & 0.38 & $60.54 \pm 8.04$ & $58.78 \pm 7.82$ & 0.353 \\
\hline Grade (\%) & & & 0.125 & & & 0.881 \\
\hline Mid-high & 43 (57.33\%) & 20 (74.07\%) & & $46(61.33 \%)$ & 17 (62.96\%) & \\
\hline Low & 32 (42.67\%) & 7 (25.93\%) & & 29 (38.67\%) & 10 (37.04\%) & \\
\hline Location (\%) & & & 0.004 & & & 0.09 \\
\hline Neck & $4(5.33 \%)$ & $1(3.70 \%)$ & & $6(8.0 \%)$ & $1(3.70 \%)$ & \\
\hline Upper-thorax & 14 (18.67\%) & $2(7.41 \%)$ & & $14(18.67 \%)$ & $6(22.22 \%)$ & \\
\hline Mid-thorax & 29 (38.67\%) & 21 (77.78\%) & & 31 (41.33\%) & 17 (62.96\%) & \\
\hline Low-thorax & 28 (37.33\%) & $3(11.11 \%)$ & & $24(32.0 \%)$ & $3(11.11 \%)$ & \\
\hline T stage $(\%)$ & & & $<0.001$ & & & 0.059 \\
\hline $\mathrm{T} 1$ & $0(0 \%)$ & $0(0 \%)$ & & $0(0 \%)$ & $0(0 \%)$ & \\
\hline $\mathrm{T} 2$ & $9(12 \%)$ & $0(0 \%)$ & & $7(9.33 \%)$ & $1(3.70 \%)$ & \\
\hline T3 & 61 (81.33\%) & 12 (44.44\%) & & 59 (78.67\%) & 17 (62.96\%) & \\
\hline $\mathrm{T} 4 \mathrm{a}$ & $0(0 \%)$ & $1(3.70 \%)$ & & $1(1.33 \%)$ & $3(11.11 \%)$ & \\
\hline $\mathrm{T} 4 \mathrm{~b}$ & $5(6.67 \%)$ & $14(51.85 \%)$ & & $8(10.67 \%)$ & $6(22.22 \%)$ & \\
\hline $\begin{array}{l}\text { Trachea } \\
\text { invasion (\%) }\end{array}$ & & & $<0.001$ & & & 0.5 \\
\hline No & 73 (97.33\%) & 15 (55.56\%) & & 67 (89.33\%) & 23 (85.19\%) & \\
\hline Yes & $2(2.67 \%)$ & 12 (44.44\%) & & $8(10.67 \%)$ & $4(14.81 \%)$ & \\
\hline $\mathrm{N}$ stage (\%) & & & 0.321 & & & 0.021 \\
\hline No & $5(6.67 \%)$ & $0(0 \%)$ & & 8 (10.67\%) & $0(0 \%)$ & \\
\hline Yes & 70 (93.33\%) & 27 (100\%) & & 67 (89.33\%) & 27 (100\%) & \\
\hline Metastasis (\%) & & & 0.387 & & & 0.55 \\
\hline No & $60(80 \%)$ & 24 (88.89\%) & & $62(82.67 \%)$ & 24 (88.89\%) & \\
\hline
\end{tabular}




\begin{tabular}{|c|c|c|c|c|c|c|}
\hline Yes & $15(20 \%)$ & $3(11.11 \%)$ & & $13(17.33 \%)$ & $3(11.11 \%)$ & \\
\hline Tumor range & & & $<0.001$ & & & 0.025 \\
\hline $1-1 / 4$ & $1(3.40 \%)$ & $0(\%)$ & & $0(0 \%)$ & $0(0 \%)$ & \\
\hline $1 / 4-1 / 2$ & $18(24.0 \%)$ & $0(\%)$ & & $11(14.67 \%)$ & $1(3.70 \%)$ & \\
\hline $1 / 2-3 / 4$ & 25 (33.33\%) & $2(7.41 \%)$ & & 30 (40.0\%) & $6(22.22 \%)$ & \\
\hline $3 / 4-1$ & 31 (41.33\%) & 25 (92.59\%) & & 34 (45.33\%) & 20 (74.07\%) & \\
\hline Type (\%) & & & 0.195 & & & 0.952 \\
\hline Focal & 17 (22.67\%) & $3(11.11 \%)$ & & 19 (25.33\%) & 7 (25.93\%) & \\
\hline Diffuse & 58 (77.33\%) & 24 (88.89\%) & & 56 (74.67\%) & 20 (74.07\%) & \\
\hline $\begin{array}{l}\text { Luminal } \\
\text { obliteration (\%) }\end{array}$ & & & 0.303 & & & 0.634 \\
\hline No & 25 (33.33\%) & 12 (44.44\%) & & 26 (34.67\%) & 8 (29.63\%) & \\
\hline Yes & 50 (66.67\%) & 15 (55.56\%) & & 49 (65.33\%) & 19 (70.37\%) & \\
\hline Deep ulcer & & & $<0.001$ & & & $<0.001$ \\
\hline No & 70 (93.33\%) & 5 (18.52\%) & & 66 (88.0\%) & 9 (33.33\%) & \\
\hline Yes & $5(6.67 \%)$ & 22 (81.48\%) & & $9(12.0 \%)$ & 18 (66.67\%) & \\
\hline THK-tumor (mm) & $14.73 \pm 4.99$ & $17.89 \pm 5.07$ & 0.006 & $15.29 \pm 6.33$ & $17.44 \pm 5.90$ & 0.118 \\
\hline L-tumor (mm) & $54.20 \pm 20.86$ & $81.67 \pm 26.19$ & $<0.001$ & $54.73 \pm 22.97$ & $75.19 \pm 28.15$ & $<0.001$ \\
\hline $\begin{array}{l}\text { THN-tumor } \\
(\mathrm{mm})\end{array}$ & $6.09 \pm 1.58$ & $10.46 \pm 6.89$ & 0.001 & $6.21 \pm 2.18$ & $4.33 \pm 1.64$ & $<0.001$ \\
\hline DEP-ulcer (mm) & $0.61 \pm 2.40$ & $10.46 \pm 6.89$ & $<0.001$ & $0.96 \pm 2.73$ & $6.59 \pm 5.73$ & $<0.001$ \\
\hline $\begin{array}{l}\text { THK-residue } \\
(\mathrm{mm})\end{array}$ & $14.16 \pm 5.20$ & $9.54 \pm 3.96$ & $<0.001$ & $13.91 \pm 6.76$ & $9.70 \pm 7.86$ & 0.002 \\
\hline $\begin{array}{l}\text { THK-adjacency } \\
(\mathrm{mm})\end{array}$ & $14.59 \pm 4.83$ & $16.54 \pm 4.96$ & 0.082 & $14.93 \pm 6.20$ & $15.63 \pm 5.85$ & 0.392 \\
\hline R-ulcer (\%) & $5.07 \pm 19.65$ & $68.98 \pm 52.67$ & $<0.001$ & $6.42 \pm 18.21$ & $46.12 \pm 37.47$ & $<0.001$ \\
\hline R-residue (\%) & $96.67 \pm 13.07$ & $43.75 \pm 28.42$ & $<0.001$ & $93.15 \pm 19.08$ & $59.01 \pm 33.54$ & $<0.001$ \\
\hline HU-min (HU) & $43.86 \pm 16.34$ & $26.33 \pm 13.55$ & $<0.001$ & $45.17 \pm 17.32$ & $30.52 \pm 13.15$ & $<0.001$ \\
\hline THK-min (mm) & $11.13 \pm 4.55$ & $13.93 \pm 4.66$ & 0.003 & $11.35 \pm 5.29$ & $12.41 \pm 5.21$ & 0.202 \\
\hline R-min (\%) & $0.77 \pm 0.19$ & $0.79 \pm 0.17$ & 0.939 & $0.75 \pm 0.17$ & $0.74 \pm 0.25$ & 0.802 \\
\hline $\mathrm{HU}-\max (\mathrm{HU})$ & $93.75 \pm 14.29$ & $90.53 \pm 12.75$ & 0.286 & $94.28 \pm 15.45$ & $85.61 \pm 12.89$ & 0.006 \\
\hline
\end{tabular}




\begin{tabular}{|c|c|c|c|c|c|c|}
\hline $\mathrm{HK}-\mathrm{max}(\mathrm{mm})$ & $11.15 \pm 3.81$ & $13.89 \pm 4.93$ & 0.01 & $10.88 \pm 3.78$ & $12.00 \pm 2.76$ & 0.068 \\
\hline R-HU (\%) & $0.47 \pm 0.17$ & $0.30 \pm 0.14$ & $<0.001$ & $0.48 \pm 0.17$ & $0.36 \pm 0.15$ & 0.002 \\
\hline \multicolumn{7}{|c|}{$\begin{array}{l}\text { Abbreviations: N stage, lymph node stage; THK-tumor, tumor thickness; L-tumor, tumor length; THN- } \\
\text { tumor, tumor thinness; DEP-ulcer, depth of deep ulcer; THK-residue, thickness of residual esophageal } \\
\text { wall in the ulcer layer; THK-adjacency, thickness of lesion adjacent to the ulcer; R-ulcer, the ulcer-to- } \\
\text { tumor ratio; R-residue, THK-residue-to-tumor ratio; HU-min, tumor minimum CT value; THK-min, } \\
\text { thickness of the tumor on minimum CT value layer; R-min, THK-min-to-THK-tumor ratio; HU-max, } \\
\text { tumor maximum CT value; THK-max, thickness of the tumor on maximum CT value layer; R-HU,HU- } \\
\text { min-to-HU-max ratio. }\end{array}$} \\
\hline
\end{tabular}

Table 2

Characteristics of patients in the test and validation cohorts 
Characteristics

Test cohort $(\mathrm{n}=102) \quad$ Validation cohort $(\mathrm{n}=102) \quad P$

\begin{tabular}{|c|c|c|c|c|}
\hline \multirow[t]{2}{*}{ Gender (\%) } & Male & 95 & 92 & 0.447 \\
\hline & Female & 7 & 10 & \\
\hline Age, mean $\pm S D$, years & & $60.21 \pm 7.84$ & $60.84 \pm 8.13$ & 0.575 \\
\hline \multirow[t]{2}{*}{ Grade (\%) } & Mid-high & 63 & 63 & / \\
\hline & Low & 39 & 39 & \\
\hline \multirow[t]{4}{*}{ Location (\%) } & Neck & 5 & 7 & 0.778 \\
\hline & Upper-thorax & 16 & 20 & \\
\hline & Mid-thorax & 50 & 48 & \\
\hline & Low-thorax & 31 & 27 & \\
\hline \multirow[t]{5}{*}{$\mathrm{T}$} & $\mathrm{T} 1$ & 0 & 0 & 0.46 \\
\hline & $\mathrm{T} 2$ & 9 & 8 & \\
\hline & T3 & 73 & 66 & \\
\hline & $\mathrm{T} 4 \mathrm{a}$ & 1 & 4 & \\
\hline & $\mathrm{T} 4 \mathrm{~b}$ & 19 & 14 & \\
\hline \multirow[t]{2}{*}{ Trachea invasion (\%) } & No & 88 & 89 & 0.836 \\
\hline & Yes & 14 & 12 & \\
\hline \multirow{2}{*}{$\mathrm{N}$ stage $(\%)$} & No & 5 & 8 & 0.39 \\
\hline & Yes & 97 & 94 & \\
\hline \multirow[t]{2}{*}{ Metastasis (\%) } & No & 84 & 86 & 0.707 \\
\hline & Yes & 18 & 16 & \\
\hline THK-tumor (mm) & & $15.57 \pm 5.18$ & $15.86 \pm 6.26$ & 0.715 \\
\hline L-tumor (mm) & & $61.47 \pm 25.37$ & $60.15 \pm 25.94$ & 0.713 \\
\hline \multirow[t]{4}{*}{ Tumor range } & $1-1 / 4$ & 1 & 0 & 0.27 \\
\hline & $1 / 4-1 / 2$ & 18 & 12 & \\
\hline & $1 / 2-3 / 4$ & 27 & 36 & \\
\hline & $3 / 4-1$ & 56 & 54 & \\
\hline \multirow[t]{2}{*}{ Type (\%) } & Focal & 20 & 26 & 0.315 \\
\hline & Diffuse & 82 & 76 & \\
\hline
\end{tabular}


Luminal obliteration (\%) No

$37 \quad 34$

$\begin{array}{lll}\text { Yes } & 65 & 68\end{array}$

THN-tumor (mm)

$5.78 \pm 1.67$

$5.71 \pm 2.20$

0.803

Deep ulcer

No

75

75

I

Yes

27

27

DEP-ulcer (mm)

$3.15 \pm 5.90$

$2.45 \pm 4.49$

0.708

THK-residue $(\mathrm{mm})$

$12.38 \pm 6.18$

$12.79 \pm 7.27$

0.948

THK-adjacency (mm)

$15.09 \pm 4.92$

$15.12 \pm 6.09$

0.566

R-ulcer (\%)

$21.52 \pm 42.05$

$16.93 \pm 30.23$

0.741

R-residue (\%)

$83.05 \pm 29.48$

$84.12 \pm 28.03$

0.827

HU-min (HU)

$39.22 \pm 17.41$

$41.29 \pm 17.51$

0.428

THK-min (mm)

$11.87 \pm 4.72$

$11.63 \pm 5.33$

0.478

R-min (\%)

$0.77 \pm 0.18$

$0.75 \pm 0.19$

0.333

HU-max (HU)

$92.90 \pm 13.91$

$91.99 \pm 15.24$

0.477

THK-max (mm)

$11.87 \pm 4.28$

$11.18 \pm 3.56$

0.442

R-HU (\%)

$0.43 \pm 0.18$

$0.45 \pm 0.17$

0.318

Abbreviations: N stage, lymph node stage; THK-tumor, tumor thickness; L-tumor, tumor length; THNtumor, tumor thinness; DEP-ulcer, depth of deep ulcer; THK-residue, thickness of residual esophageal wall in the ulcer layer; THK-adjacency, thickness of lesion adjacent to the ulcer; R-ulcer, ulcer-to-tumor ratio; R-residue, THK-residue-to-tumor radio; HU-min, tumor minimum CT value; THK-min, thickness of the tumor on minimum CT value layer; R-min, THK-min-to-THK-tumor radio; HU-max, tumor maximum CT value; THK-max, thickness of the tumor on maximum CT value layer; R-HU,HU-min-to-HU-max ratio

\section{Table 3}

\section{Logistic regression analysis of quantitative variables in the test cohort}

\begin{tabular}{lllllll} 
Variables & B & OR & $95 \% \mathrm{Cl}$ & $P$ & AUC & Cutoff \\
THK-tumor & 0.155 & 1.167 & $1.009-1.351$ & 0.037 & $0.696(0.582-0.809)$ & 15.5 \\
\hline R-ulcer & 5.106 & 164.947 & $20.464-1329.511$ & $<0.001$ & $0.887(0.800-0.975)$ & 0.18 \\
R-HU & -5.105 & 0.006 & $0.001-0.782$ & 0.039 & $0.774(0.677-0.872)$ & 0.37
\end{tabular}

Abbreviations: THK-tumor, tumor thickness; R-ulcer, ratio of deep ulcer; R-HU, HU-min-to-HU-max ratio; $B$, regression coefficient; OR, odds ratio; $95 \% \mathrm{Cl}$, 95\%confidence interval; AUC, area under the curve. 


\section{Table 4}

\section{Logistic regression analysis of quantitative variables in the test and validation cohorts}

\begin{tabular}{|c|c|c|c|c|c|c|c|c|}
\hline Cohorts & Variables & AUC & Cutoff & SEN & SPE & PPV & NPV & $\mathrm{ACU}$ \\
\hline \multirow[t]{2}{*}{ Test cohort } & Y1 & $\begin{array}{l}0.946(0.902- \\
0.990)\end{array}$ & 2.1 & 0.885 & 0.933 & 0.828 & 0.959 & 0.922 \\
\hline & Y2 & $\begin{array}{l}0.953(0.909- \\
0.997)\end{array}$ & 2.58 & 0.923 & 0.947 & 0.862 & 0.973 & 0.941 \\
\hline \multirow[t]{2}{*}{$\begin{array}{l}\text { Validation } \\
\text { cohort }\end{array}$} & Y1 & $\begin{array}{l}0.841(0.758- \\
0.924)\end{array}$ & 2.1 & 0.63 & 0.84 & 0.586 & 0.863 & 0.784 \\
\hline & Y2 & $\begin{array}{l}0.917(0.864- \\
0.969)\end{array}$ & 2.58 & 0.63 & 0.827 & 0.567 & 0.861 & 0.775 \\
\hline
\end{tabular}

Abbreviations: $\mathrm{Y} 1=$ joint predictive efficiency of quantitative $\mathrm{CT}$ analysis; $\mathrm{Y} 2=$ joint predictive efficiency of quantitative CT analysis added with T stage; AUC, area under the curve; SEN, Sensitivity; SPE, Specificity; PPV, Positive predict value; NPV, Negative predict value; ACU, Accuracy.

\section{Table 5}

Efficacy of qualitative variables in predicting esophageal fistula in the test cohort.

\begin{tabular}{llllllll} 
& AUC & Cutoff & SEN & SPE & PPV & NPV & ACU \\
\hline Trachea invasion & $0.709(0.579-0.838)$ & Present & 0.444 & 0.973 & 0.857 & 0.839 & 0.833 \\
\hline N stage & $0.533(0.410-0.657)$ & Present & 1 & 0.067 & 0.278 & 1 & 0.314 \\
\hline Tumor range & $0.756(0.660-0.853)$ & $3 / 4-1$ & 0.926 & 0.587 & 0.49 & 0.973 & 0.676 \\
\hline T stage & $0.726(0.600-0.852)$ & T4b & 0.519 & 0.933 & 0.737 & 0.843 & 0.824 \\
\hline Location & $0.696(0.582-0.809)$ & Mid-thorax & 0.778 & 0.613 & 0.885 & 0.42 & 0.657 \\
\hline Deep ulcer & $0.874(0.782-0.966)$ & Present & 0.815 & 0.933 & 0.815 & 0.933 & 0.902
\end{tabular}

Abbreviations: AUC, area under the curve; SEN, Sensitivity; SPE, Specificity; PPV, Positive predict value; NPV, Negative predict value; ACU, Accuracy; P, positive; N stage, lymph node stage.

\section{Figures}


Study flowchart. SCC: squamous cell carcinoma, CRT: chemoradiotherapy.

Figure 2

Schematic diagram of esophageal carcinoma range and measurement of a deep ulcer.

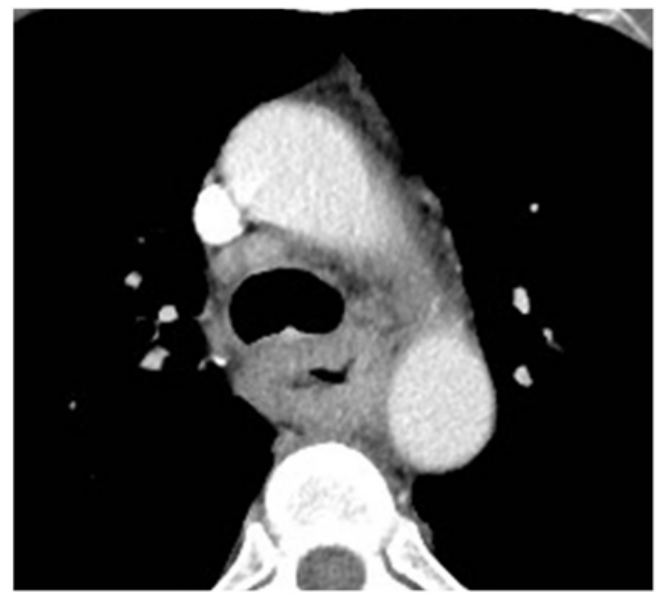

A

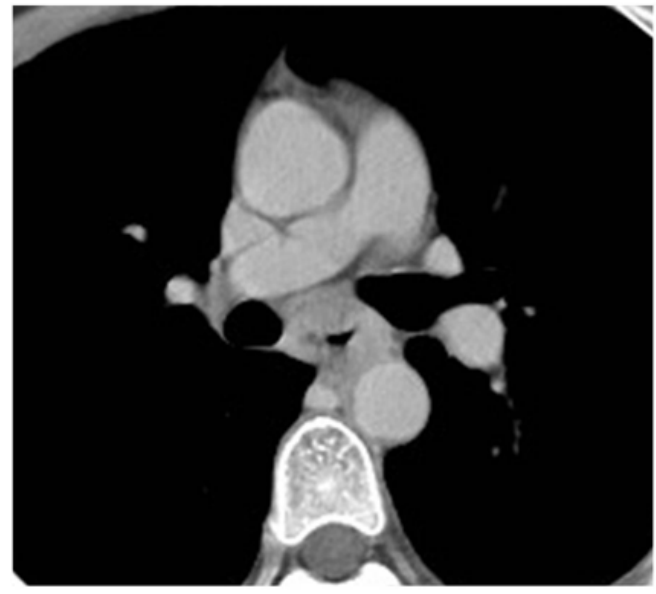

D

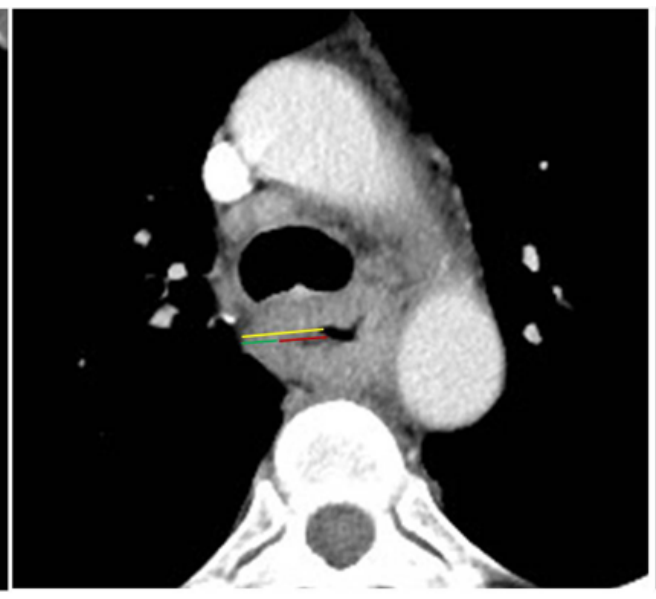

B

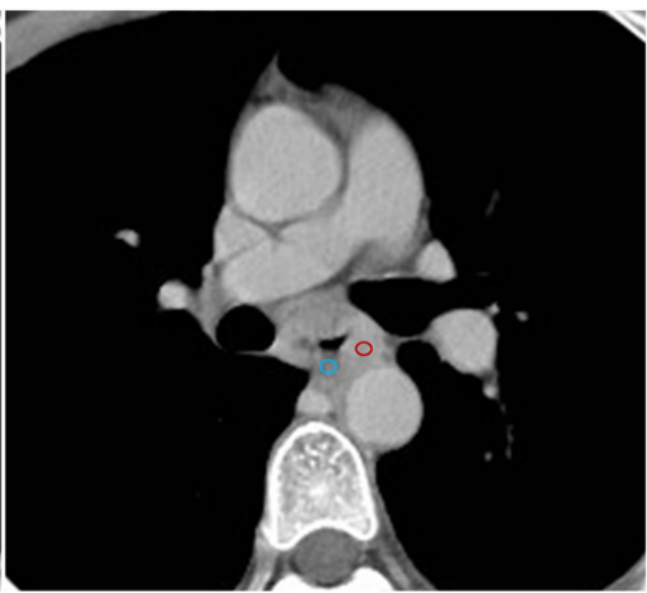

E

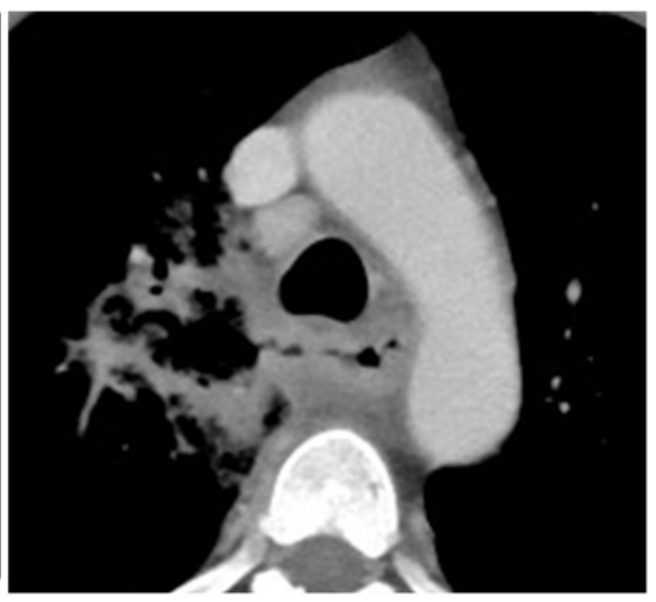

C

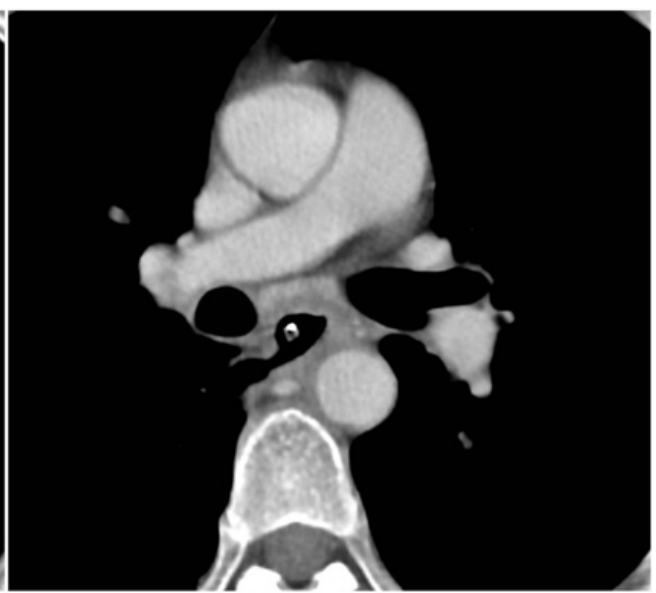

$\mathrm{F}$

\section{Figure 3}

Axial enhanced CT at baseline examination showing ROI delineation and deep ulcer measurement.

(A-C) CT images in a 42-year-old woman with esophageal cancer. (A) CT image showing an esophageal mass with a deep ulcer in the right wall. (B) The same CT image showing the measurement of the depth of deep ulcer (red line) at $10 \mathrm{~mm}$, residual esophageal wall (green line) at $7 \mathrm{~mm}$ and adjacent lesion (yellow line) at $17 \mathrm{~mm}$ in the ulcer layer; the ratio of ulcer depth to adjacent tumor thickness of $58.8 \%$ and tumor thickness of $17 \mathrm{~mm}$ could predict fistula formation after treatment. (C) The esophageal fistula occurred in the region of the deep ulcer after three chemotherapy cycles and radiotherapy. (D-F) CT images in a 50-year-old man with esophageal cancer. (D) CT image showed an esophageal mass with a thickness of $15 \mathrm{~mm}$. (E) The highest (red circle; $119 \mathrm{HU}$ ) and lowest enhancement (blue circle; $20 \mathrm{HU}$ ) 
zones, the ratio of minimum to maximum enhanced CT value of $16.8 \%$ and a tumor thickness of $15 \mathrm{~mm}$ could predict fistula formation after treatment. (F) Esophageal fistula occurred in the region of the lowest zone after three chemotherapy cycles and radiotherapy.

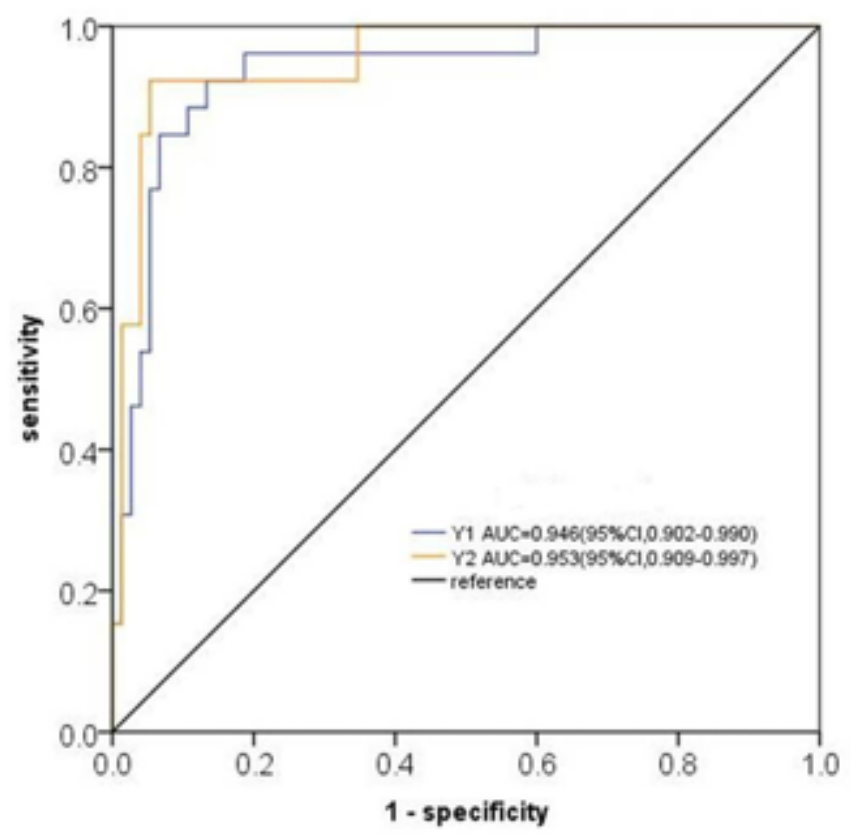

A

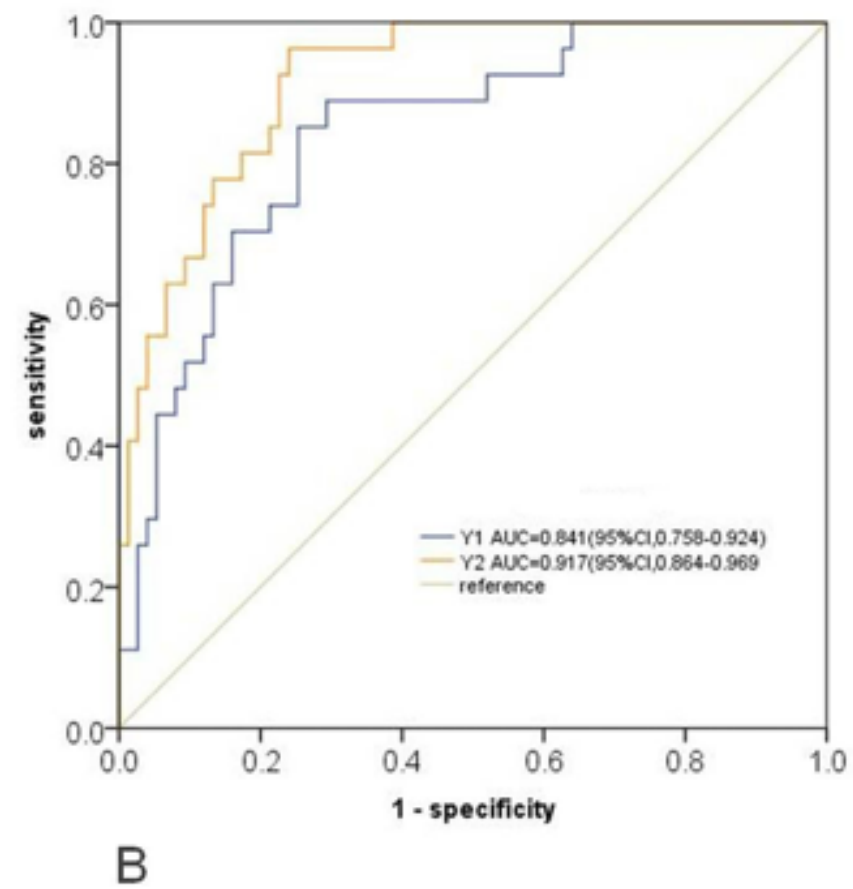

Figure 4

ROCs of baseline CT for predicting esophageal fistula in the test and validation cohorts.

The blue line (Y1) depicts the predictive performance of quantitative CT analysis. The yellow line (Y2) depicts the performance of quantitative analysis combined with $\mathrm{T}$ stage. The AUCs for $\mathrm{Y} 1$ and $\mathrm{Y} 2$ were 0.946 and 0.953 in the test cohort (A) and 0.841 and 0.917 in the validation cohort (B), respectively (both $p>0.05)$. 
Points

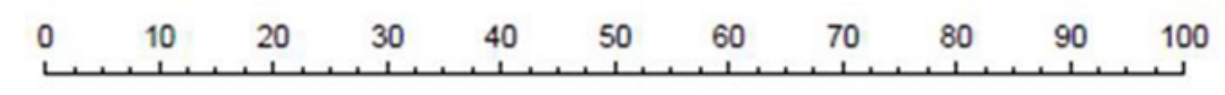

\section{THK-tumor $\begin{array}{lllllllll}6 & 1 & 1 & 1 & 1 & 1 & 1 & 1 & 1\end{array}$}

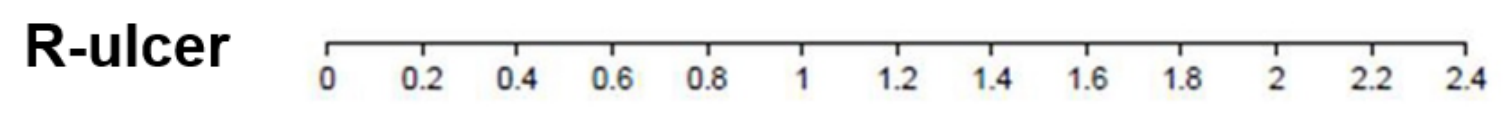

R-HU

$\begin{array}{lllll}0.9 & 0.7 & 0.5 & 0.3 & 0.1\end{array}$

\section{Total Points}

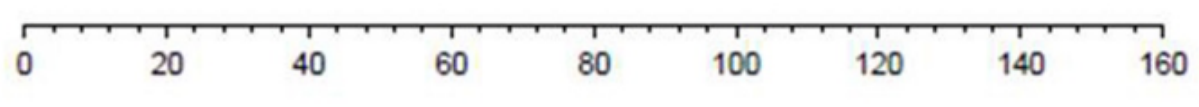

Risk

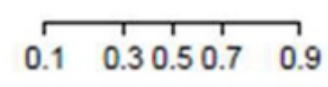

Figure 5

A nomogram established using quantitative CT parameters. 
Nomogram presents a scoring model using three quantitative CT features, including tumor thickness (THK-tumor), the ulcer-to-tumor ratio (R-ulcer), and HU-min-to-HU-max ratio (R-HU). 